\title{
Differentiating the core machinery
}

For almost 30 years, the core transcription machinery has been thought of as an invariant and universal entity in eukaryotic cells. New findings now overturn this concept, showing that an alternative version of this machinery has an essential role in the differentiation of muscle cells.

In the traditional view of the core transcription machinery, the TATAbinding protein (TBP) and the TFIID complex are invariant components.

Previous studies have highlighted the existence of factors that are related to TBP, and have also uncovered alternative, celltype-specific components of the TFIID complex.

However, the accepted thinking was still that TBP and some form of TFIID are essential for transcription.

Deato and Tjian looked at the expression of one alternative TFIID subunit,
TAF3, in a muscle precursor (myoblast) cell line and in cells taken from skeletal muscle tissue. In both cases, the standard TFIID subunits and TAF3 were shown to be expressed in myoblasts. Surprisingly, however, all of the standard TFIID components tested were dramatically downregulated in differentiated cell types (myotubes and myofibres). These results raised an exciting possibility: the TFIID complex might be lost during differentiation, with alternative components - including TAF3 - taking over the task of recognising core promoter sequences to direct the expression of muscle-specific genes.

How might TAF3 - which itself has no sequence-specific DNA binding ability — recognise promoter sequences? Deato and Tjian showed that a TBP-related protein, TRF3, has a similar expression profile to TAF3 during muscle differentiation, suggesting that the two might interact and replace the canonical TFIID complex. In support of this, the authors showed that TAF3 and TRF3 exist in a complex in vivo, and interact directly in in vitro binding studies.
Does the TAF3-TRF3 complex have functional relevance? In the myoblast cell line, knockdown of either TAF3 or TRF3 using RNAi resulted in differentiation defects - no myotubes were formed and key regulators of muscle differentiation failed to be expressed. Using chromatin immunoprecipitation, the authors showed that the core promoter of one of these regulators - the transcription factor myogenin - is highly enriched for TAF3 and TRF3 specifically in differentiated cells. Intriguingly, TBP and components of the core TFIID complex were found to be present in the RNAi-treated cells, revealing that the 'standard' transcriptional core machinery isn't sufficient to support the transcription of muscle-specific genes.

The authors speculate that this use of alternative transcriptional machinery in differentiated muscle cells might allow a rapid switch between transcriptional programmes. Investigating whether a similar process happens in the differentiation of other cell types will be an obvious goal for future studies.

Louisa Flintoft

ORIGINAL RESEARCH PAPER Deato, M.D. E. \&

Tjian, R. Switching of the core transcription machinery during myogenesis. Genes Dev.

17 August 2007 (doi: 10.1101/gad.1583407) WEB SITE

Robert Tjian's laboratory:

http://mcb.berkeley.edu/faculty/BMB/tjianr.html

\section{RESEARCH HIGHLIGHTS ADVISORS}

MICHAEL AKAM

University of Cambridge, UK SEAN B. CARROLL

University of Wisconsin, USA NANCY J. COX

University of Chicago, USA

SUSAN FORSBURG University of

Southern California, USA
RALPH J. GREENSPAN

The Neurosciences Institute, California, USA YOSHIHIDE HAYASHIZAKI Riken Genomic Sciences Center, Japan MARK JOBLING

University of Leicester, UK
PETER KOOPMAN

University of Queensland, Australia LEONID KRUGLYAK Fred Hutchinson Cancer Research Center, USA BARBARA MEYER

University of California, Berkeley, USA
JOHN QUAKENBUSH

Dana-Farber Cancer Institute and Harvard School of Public Health, Boston, USA JANET ROSSANT

Mount Sinai Hospital, Toronto, Canada MARC VIDAL Dana-Farber Cancer Institute, Boston, USA
VIRGINIA WALBOT

Stanford University, USA DETLEF WEIGEL Max Planck Institute for Developmental Biology, Germany PHIL ZAMORE

University of Massachusetts, USA LEONARD I. ZON

Children's Hospital, Boston, USA 